\title{
Low Back Pain: A Pain That May Not Be Harmless
}

\author{
Catarina Lucas, Joana Costa, Joana Paixão, Fátima Silva, Pedro Ribeiro, Adriano Rodrigues \\ Serviço de Medicina Interna B, Hospital Geral, Centro Hospitalar e Universitário de Coimbra, Coimbra, Portugal
}

How to cite this article: Lucas C, Costa J, Paixão J, Silva F, Ribeiro P, Rodrigues A. Low back pain: a pain that may not be harmless. EJCRIM 2018;5: doi:10.12890/2018_000834.

Conflicts of Interests: The Authors declare that there are no competing interests.

This article is licensed under a Commons Attribution Non-Commercial 4.0 License

\section{ABSTRACT}

Abdominal aortic aneurysms are defined as vascular dilatations greater than $50 \%$ of the normal proximal segment or those that have a maximum diameter above $3 \mathrm{~cm}$. Risk factors include male gender, age over 75 years, history of vascular pathology, hypertension and arteriosclerosis. The Authors describe a case of a 74-year-old man, evaluated in an emergency setting for right lumbar pain lasting 4 days. The pain did not respond to analgesia and became progressively worse. Due to the severity of symptoms, CT angiography was performed, which showed an active rupture of a partially contained aneurysm associated with aortic dissection. Early diagnosis and timely management of aortic aneurysms are essential in preventing complications, namely rupture (50-83\% patients die after rupture and before receiving medical care). Acute aortic dissection is a surgical emergency and the risk of rupture is proportional to the size of the aneurysm and its rate of growth.

\section{LEARNING POINTS}

- This article shows how difficult it is sometimes to make the right diagnosis in an emergency department.

- Although a very common symptom, low back pain should not be regarded as harmless.

\section{KEYWORDS}

Abdominal aneurysm, vascular aneurysm, aortic aneurysm, iliac artery aneurysm, aortic rupture, lumbar pain, low back pain

\section{INTRODUCTION}

Abdominal aortic aneurysm is a pathology associated with advanced age, the diagnosis being made mainly in individuals over 65 years ${ }^{[1,2]}$. As the aneurysm diameter increases (especially for values greater than $3 \mathrm{~cm}$ ), the risk of rupture is greater, often with a fatal outcome ${ }^{[2,3]}$. The vast majority of abdominal aortic aneurysms are diagnosed incidentally, and are often attributable to atherosclerotic disease of the aorta ${ }^{[1]}$. In these patients, early detection of aneurysm rupture is critical and surgical intervention a priority.

\section{CASE DESCRIPTION}

The Authors describe the case of a 74-year-old man with a history of hypertension, obesity, dyslipidaemia, hyperuricemia, and cardiac and renal vascular pathology. He was taking oral antidiabetic and antihypertensive medications, among others. He was evaluated in an emergency context due to lumbar pain radiating to his right flank, lasting 4 days.

At initial presentation in the emergency department, urinary infection was excluded because of the location of the pain. Given the suspicion of renal colic, an abdominal ultrasound was performed, which did not confirm colic but showed the presence of aneurysms, dependent on the abdominal aorta, at the bifurcation of the right common iliac artery; both aneurysms were about $6 \mathrm{~cm}$ in diameter. However, there was 
no evidence of possible haematoma or rupture.

Following evaluation by Orthopaedics, acute pathology in degenerative lesions at the lumbar spine level was excluded. The patient remained on surveillance for lumbar pain, despite escalating analgesic therapy. During his hospital stay, he experienced two febrile peaks, with leucocytosis $\left(16,000 / \mathrm{mm}^{3}\right)$ and elevated C-reactive protein $(7.6 \mathrm{mg} / \mathrm{dL})$. Septic screening was performed, delaying the introduction of antibiotic therapy. After about 12 hours, the patient experienced considerable worsening of his low back pain associated with fever and hypertensive peak (BP 186/64 mmHg, similar in both limbs). He was unable to tolerate the dorsal decubitus position and had very limited improvement in pain following intravenous pethidine and also morphine. Considering the deterioration of his clinical condition, CT angiography was performed to exclude complications, namely aneurysm rupture (Fig. 1, arrow). CT angiography showed a right common iliac artery aneurysm, where the artery bifurcates into the internal and external iliac arteries; and an intramural haematoma was also observed, with signs of active bleeding in the anterior strand. There was also densification of adjacent retroperitoneal fat. The patient underwent emergent surgery with a positive outcome.

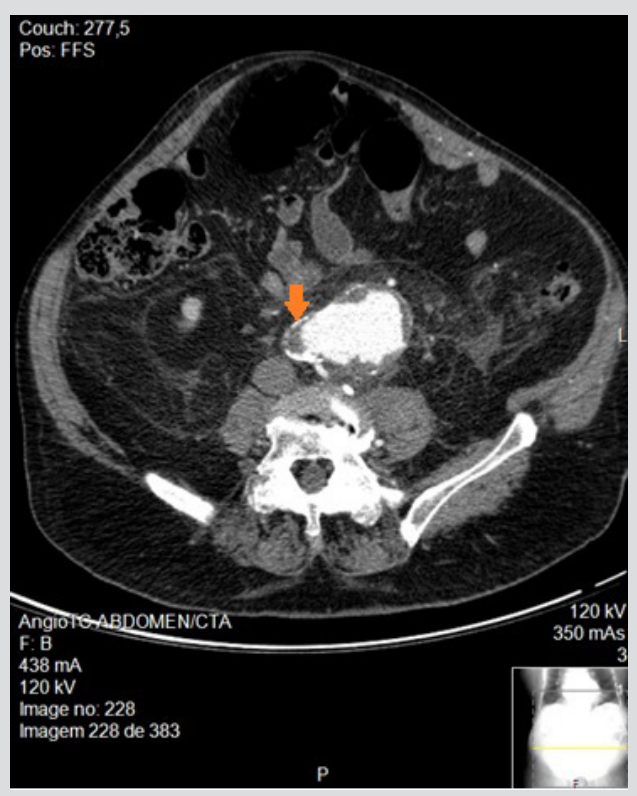

Figure 1. CT angiography showing a right common iliac artery aneurysm (with arrow), where the artery bifurcates into the internal and external iliac arteries, and also an intramural haematoma

\section{DISCUSSION}

Aortic aneurysm rupture is a relatively frequent cause of intra-hospital and extra-hospital mortality ${ }^{[2,3]}$. The most frequent location of the rupture is at the infrarenal level, extending to the iliac arteries ${ }^{[2]}$. The triad of acute abdominal or lumbar pain, hypotension and shock is the most usual clinical presentation ${ }^{[1-3]}$. In the described case, the classical presentation was not present. It may be attributable to a possible primary rupture with a contained haematoma, which, on the one hand, masked the extent of the symptoms, but, on the other hand, may have contributed to the relative clinical stability of the patient, thus avoiding a fatal outcome.

\section{CONCLUSION}

The purpose of this case description is to emphasise the need for a thorough approach to low back pain, despite its aetiological diversity. It deserves to be high in the differential diagnosis of abdominal aortic aneurysm, particularly when there is a poor response to optimised analgesic therapy. Situations with a high mortality rate, such as a ruptured aortic aneurysm, may therefore be diagnosed at an early stage, resulting in a significant improvement in prognosis.

\section{REFERENCES}

1. Kumar Y, Hooda K, Li S, Goyal P, Gupta N, Adeb M. Abdominal aortic aneurysm: pictorial review of common appearances and complications. Ann Transl Med 2017:5:256.

2. Levy D, Le JK. Aortic, dissection. StatPearls [Internet]. Treasure Island, FL: StatPearls Publishing, 2017 Jun. Available from: https://www.ncbi.nlm.nih.gov/books/NBK441963/

3. Keisler B, Carter C. Abdominal aortic aneurysm. Am Fam Physician 2015;91:538-43. 